\title{
Development of a Multibody Systems Model for Investigation of the Effects of Hybrid Electric Vehicle Powertrains on Vehicle Dynamics.
}

\author{
http://dx.doi.org/10.3991/ijoe.v11i6.5033 \\ Matthew Bastin* and R Peter Jones \\ University of Warwick, Coventry, UK
}

\begin{abstract}
With ever increasing numbers of Hybrid Electric Vehicles (HEV's) being developed, come new challenges in the field of automotive engineering. Whilst there has been considerable work conducted on HEV's from a powertrain, efficiency, and control systems perspective, very little work has been instigated in the field of how the introduction of such hybrid systems effect passive vehicle dynamics. One of the possible obstacles in the way of such studies is the multitude of powertrain architectures that are present or possible in HEV's. This obstacle can make investigations very application specific, and leads to inefficiencies in the modelling process.
\end{abstract}

This paper discusses the development of a model constructed in Dymola in order to investigate the effects of hybrid powertrains on ride and handling. The modelling methodology is presented, along with model based testing and validation of component and the full vehicle models.

Whilst the development of the model is introduced for a specific study, it is shown that the way in which the model has been developed lends itself easily to use in other fields. It is shown that the modular construction of the model, and the physical, object orientated modelling approach facilitated by Dymola, allow varying numbers and complexities of component models to be utilised within the same basic model. Such an approach means that one base model can be utilised for differing hybrid architectures for ride, handling and drivability studies thus reducing modelling time and complexity.

Index terms-Multibody Modelling, Systems Modelling, Dymola, Ride, Handling, Vehicle Dynamics

\section{INTRODUCTION}

This paper will present an approach for creating a multibody chassis model to be used for studies relating primarily to vehicle ride (primary) and handling of Hybrid Electric Vehicles (HEV's) [1] [2]. It will be shown that utilising the Dymola modelling packages that such models can be created in a modular fashion, which can be used to facilitate the easy adaption of the model from one hybrid architecture to another. It will also be discussed how the model complexity can be easily added to, or reduced to investigate primary and secondary ride, handling and driveability.

\section{Modelling Methodology}

Utilising the multi-domain, object orientated, physical modelling approach facilitated by Dymola, can allow the inclusion of mechanical, electrical, thermal and control models in one modelling domain, making it ideally suited for modelling HEV's. Further to this the models are physically based on real systems making them inherently easier to understand, and simple models can be aggregated into single components allowing them to be efficiently reused.

The model developed here makes use of the majority of these points. Chassis systems are built and validated at a component level, and then modularly constructed to produce a full vehicle model.

The model presented here was created for use in a study that investigated the ride and handling of a conventionally powered vehicle and a hybrid vehicle based on the same platform. As such specific detail was given to suspension models as well as detailed mass and inertia models of the vehicle body. Engine and drivetrian models are of basic complexity as they were not of critical importance for the specific study.

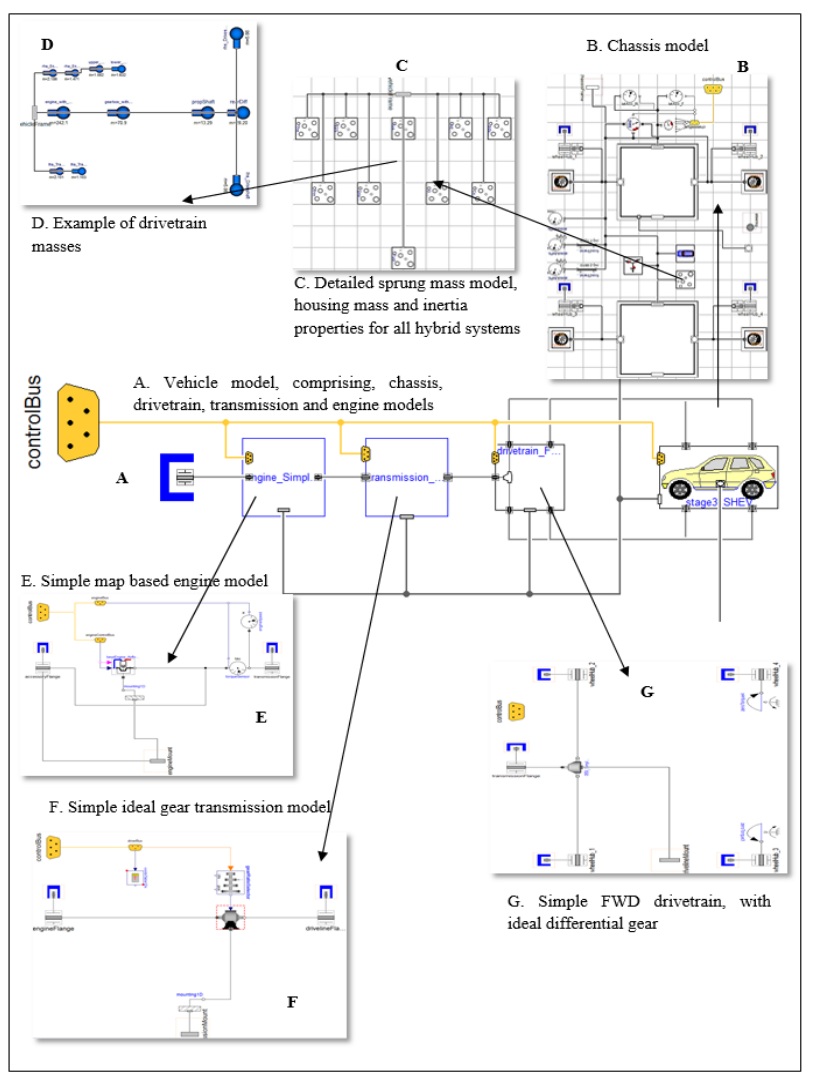

Figure 1. Vehicle chassis model construction 
As the mass and inertia properties of HEV's play such a large roll in dictating their ride and handling, masses of components in the conventional vehicle that were to be removed in creating the hybrid vehicle were modelled separately as multibody masses. By modelling in this way it allows the mass model of the vehicle to be quickly and accurately updated when changing from the conventional to the hybrid vehicle. It also allows the mass and inertia properties to be accurately updated for any hybrid architecture, as components are physically added and removed from the model as in reality.

\section{A. Suspension and Chassis Models}

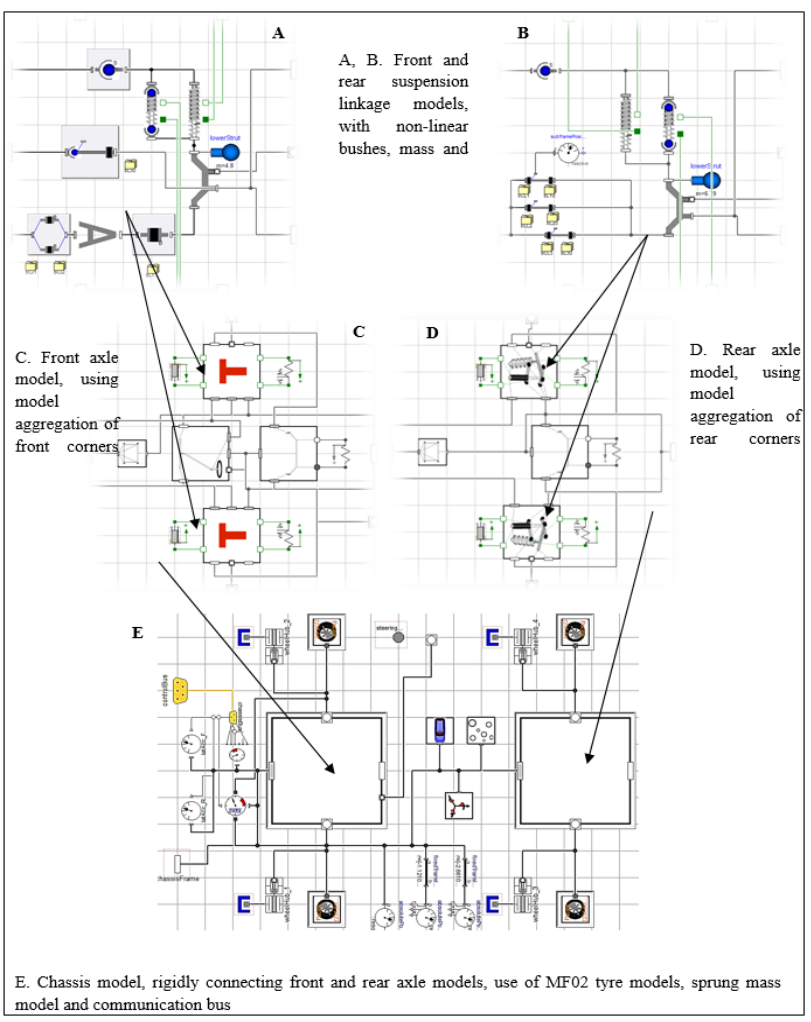

Figure 2. Vehicle model construction

\section{B. Vehicle Model}

Figure 3 shows the vehicle model introduced in this section interfaced with a driver, road and environment models.

Within the Modelon vehicle dynamics library for Dymola there are a large number of driver models, ranging from completely open loop (as shown in the figure) to completely closed loop, all of which can be utilised with the model outlined in this section.

Within the specific study that this model was utilised for a number of road models were required. Ranging from circular path models to obtain steady state characteristics to rough road models to investigate ride domain responses. All requirements could be achieved by utilising the 'Road Builder' function that is in the Modelon library. This function allows a large variety of road surfaces to be utilised with vehicle models such as this one. Exampled an uneven and circular road are given in Figure 4.

\section{MODEL VALIDATION}

Validation of the model consisted of two stages, firstly component models were validated through the use of

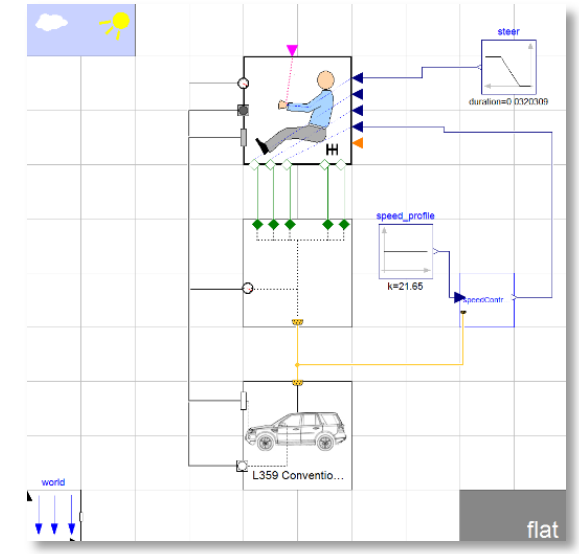

Figure 3. Vehicle model interface with driver model

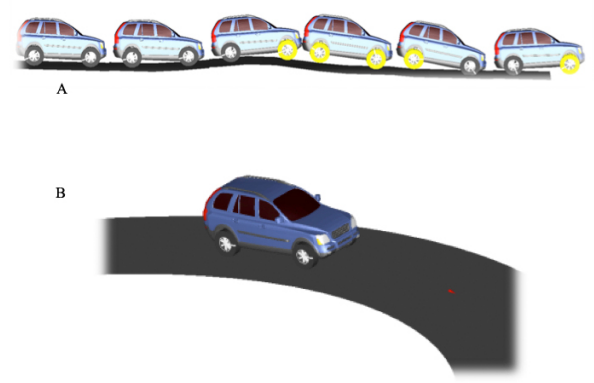

Figure 4. Vehicle and road simulation examples: A: uneven road - B: circular road

model based test rigs, once satisfactory validation at this level had been achieved then model validation was undertaken at a top level.

\section{A. Component Level Validation}

Within the Modelon Vehicle Dynamics Library for Dymola there are a number of pre-defined model based test rigs. Such models allow the mounting of suspension linkages, axles and even full vehicles (in the case of 4/7post rigs).

In this instance, corner and axle models were used with such test rigs in order to obtain kinematic data, this allowed comparison between kinematic metrics obtained from the modelling domain and those obtained from real world tests on a kinematics and compliance $(\mathrm{K} \& \mathrm{C})$ rig. Figure 5 shows the model setup for the front axle. Motions, forces and moments can be applied to the hubs as inputs and corresponding motions, force and moments in all 3 orthogonal axes obtained.

An example of the kinematic results that can be obtained are shown in Figure 7, and metrics used for correlation to test data are shown in Table 2 . It can be seen that the two sources of kinematics data agreed very well.

To validate parameters of the chassis model, an inertia rig was modelled as shown in Figure 6. This allowed the vehicle to be mounted with its Cog coaxial to the rotation axis of the rig in all 3 axes, with at torque applied to the rig, the pitch, roll and yaw inertia of the complete vehicle could be obtained. Comparison between this data obtained in the modelling domain and OEM estimates (as shown in Table 1) increases confidence in model accuracy, especially the detailed mass model. 


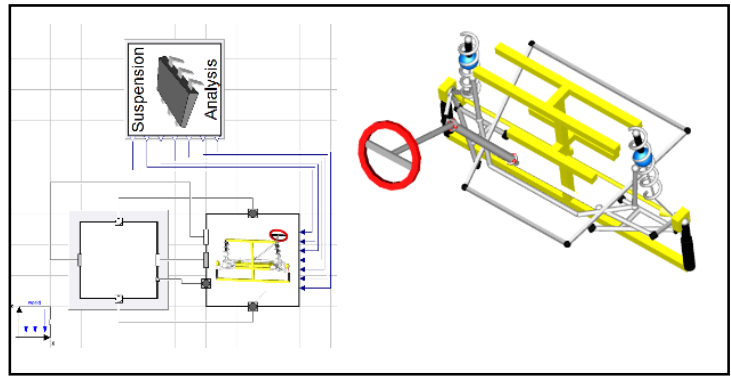

Figure 5. Model based rig

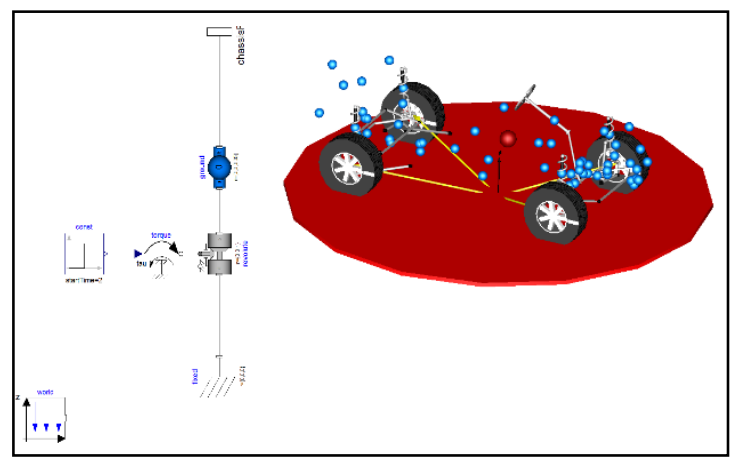

Figure 6. Model based inertie rig
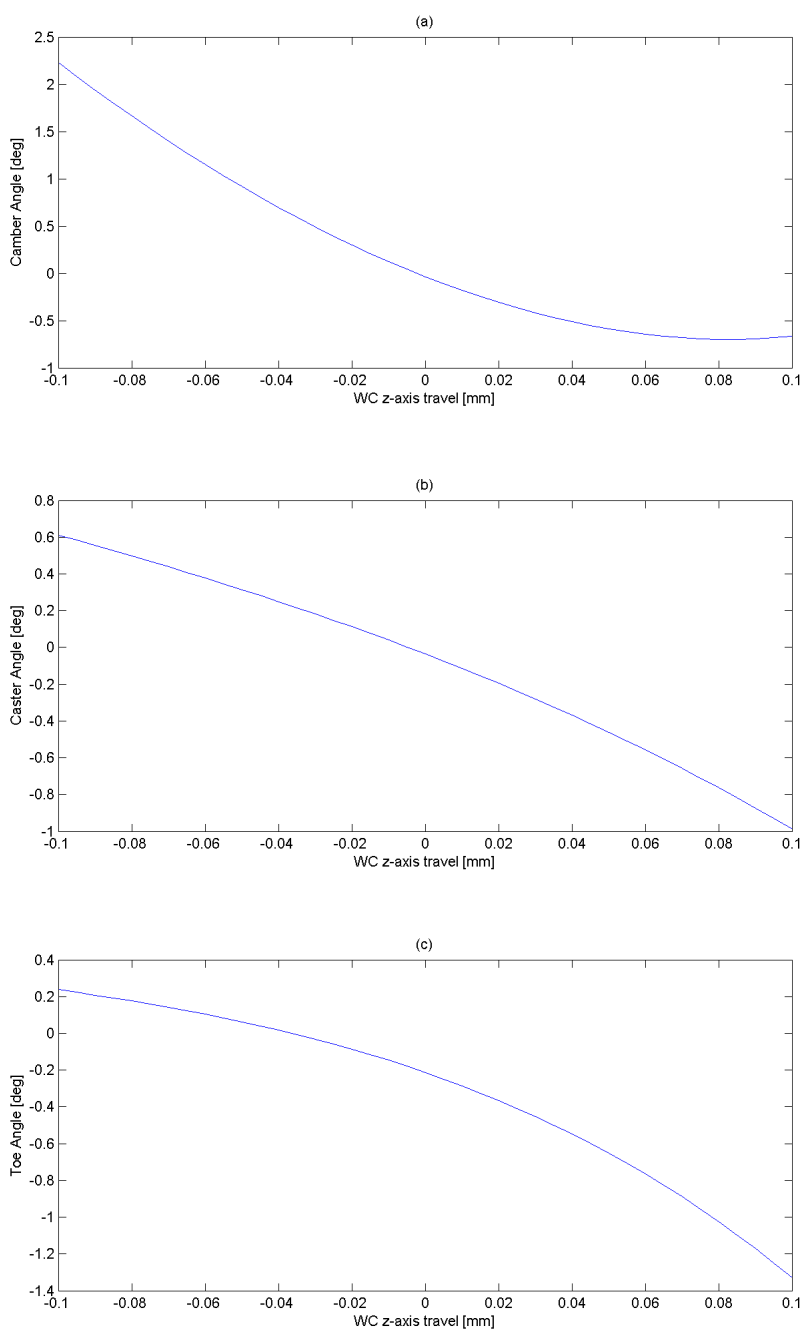

Figure 7. Caster camber and toe curves from model based rig
TABLE I.

INERTIAL DATA VALIDATION

\begin{tabular}{llcc}
\hline Metric & Unit & Dymola Model & OEM Estimate \\
Vehicle Roll Inertia & $\mathrm{Kg} \cdot \mathrm{m} 2$ & 859.4 & 815.5 \\
Vehicle Pitch Inertia & $\mathrm{Kg} \cdot \mathrm{m} 2$ & 3471.7 & 3365.0 \\
Vehicle Yaw Inertia & $\mathrm{Kg} \cdot \mathrm{m} 2$ & 3776.5 & 3720.0 \\
\hline
\end{tabular}

TABLE II.

KINEMATIC DATA VALISDATION

\begin{tabular}{llrrrrr|}
\hline \multirow{2}{*}{ Metric } & \multicolumn{2}{c}{ Dymola Model } & \multicolumn{2}{c}{ OEM Data } \\
\cline { 2 - 7 } & Unit & \multicolumn{1}{c}{ Front } & \multicolumn{1}{l}{ Rear } & \multicolumn{1}{l}{ Front } & \multicolumn{1}{l}{ Rear } \\
Camber gain & deg $/ \mathrm{m}$ & -13.67 & -16.77 & 13.00 & 16.00 \\
Bump steer & $\mathrm{deg} / \mathrm{m}$ & 7.60 & -0.04 & 7.4 & -0.05 \\
Motion ratio & $\mathrm{m} / \mathrm{m}$ & 0.95 & 0.95 & 0.95 & 0.95 \\
Wheel centre recession & $\mathrm{m} / \mathrm{m}$ & -0.01 & 0.15 & -0.01 & 0.16 \\
Static KPI & $\mathrm{deg}$ & 13.1 & - & 13.1 & - \\
Caster Trail & $\mathrm{mm}$ & 25 & - & 25 & - \\
\hline
\end{tabular}

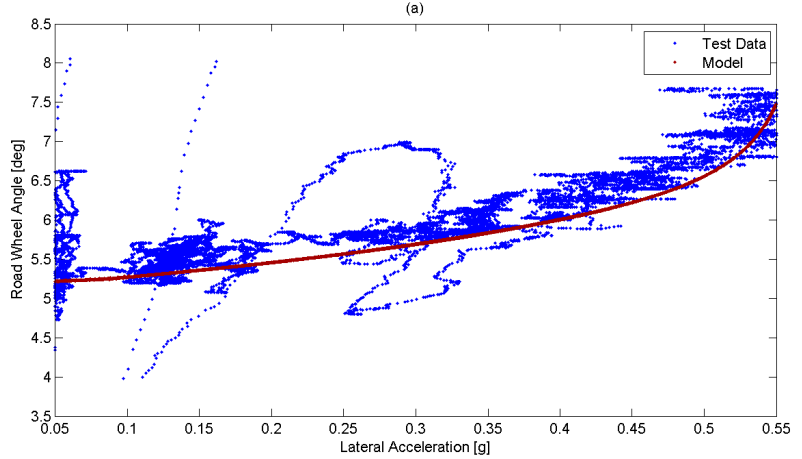

Figure 8. Steering response, real data vs. model

\section{B. Full Model Validation}

In order to validate the complete vehicle model in the handling domain a number of steady state and transient manoeuvres were conducted with a test vehicle of the same type as that modelled.

To illustrate the steady state correlation an example of a steering response plot is shown in Figure 8. The same manoeuvre was carried out in the modelling domain by interfacing the vehicle model with a circular road model produced via the road builder function of the Modelon vehicle dynamics library and a lateral closed loop driver model which could perform route following. Longitudinal control was left open loop to allow for the vehicle speed to be gradually increased.

The model data shown in Figure 8 agrees well with the collected test data, both the undertseer gradients (gradient of plot) and limit conditions correlate well between the two sets of data.

To correlate the dynamic responses a number of sinusoidal and ramp-to-step steer manoeuvres were conducted with the same test vehicle. Here to correlate to model data, all model inputs were open loop and the model was simply fed with the speed and steering profiles from the test vehicle. An example of some sinusoidal steer manoeuvre results are shown in Figure 9. It is again seen that the model outputs agree well with the test data.

\section{Model UsAGE}

The specific model constructed within this paper was utilised in a study that compared the ride and handling of a conventionally powered vehicle and its hybrid counter- 
PAPER

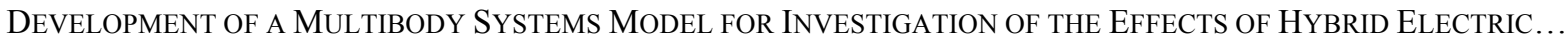
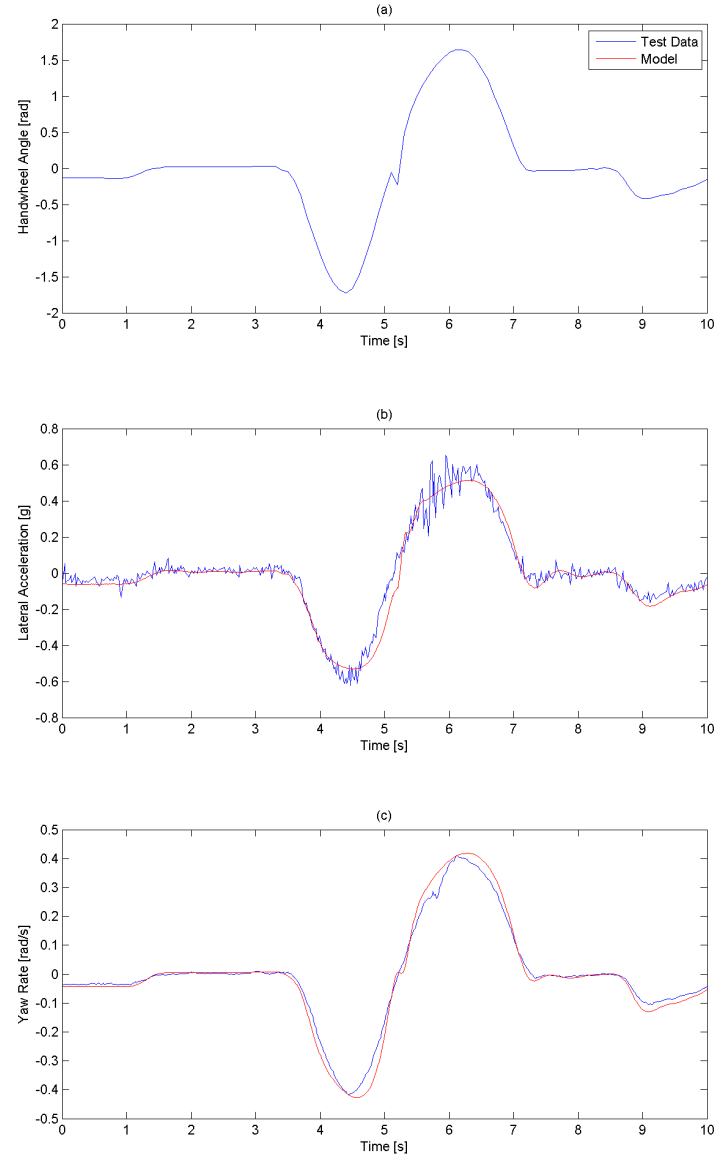

Figure 9. Lateral acceleration and yaw rate responses, real data vs model

part. The process of creating and validating the conventional model has been outlined in previous sections.

Alteration of the aforementioned conventional vehicle model to create the hybrid vehicle model was made easy through the object orientated modelling approach. For example to update the mass and inertia properties of the vehicle, a different instance of the mass and inertia sub model was created that housed data for the additional components of the hybrid vehicle. This model was then simply used to replace the conventional vehicle mass and inertia sub model. Likewise, all changes that had to be made to the conventional vehicle model to create the hybrid model, were made at a component level by simply creating a new instance of the conventional sub model. Conventional and hybrid models are then saved in a hierarchical library and can be swapped in and out of any model of the same format illustrated here.

\section{A. Model Extension}

The modelling architecture outlined here can easily be extended through approaches discussed in the previous section to suit any variant of hybrid architecture, for ride, handling and drivability studies. Powertrain and drivetrain models can updated/replaced with models that have representations of electric motor drive and compliances, as shown in Figures 10 to 12. Multiple electric motor models could be utilised and through different transmissions models can be implemented in series, parallel and series parallel configurations. Such models could prove vital for use in ride and drivability studies of current and future hybrid vehicles

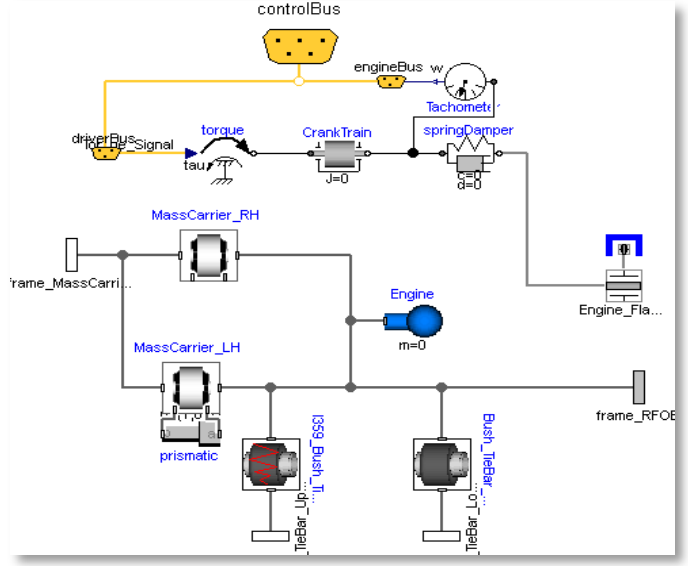

Figure 10. Compliantly mounted engine [3]

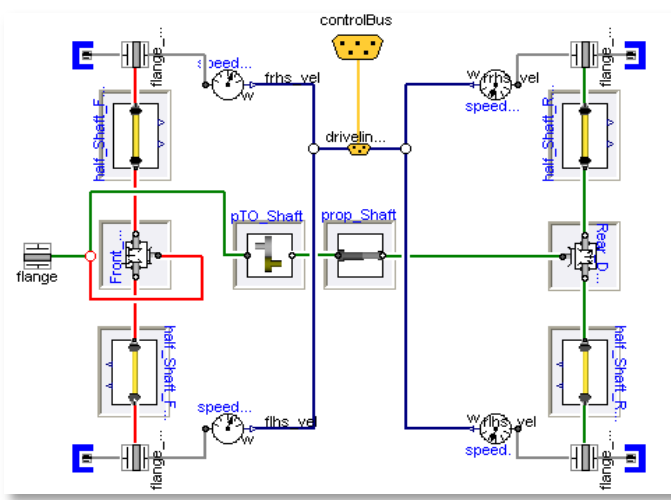

Figure 11. Rigid 4wd driveline [3]

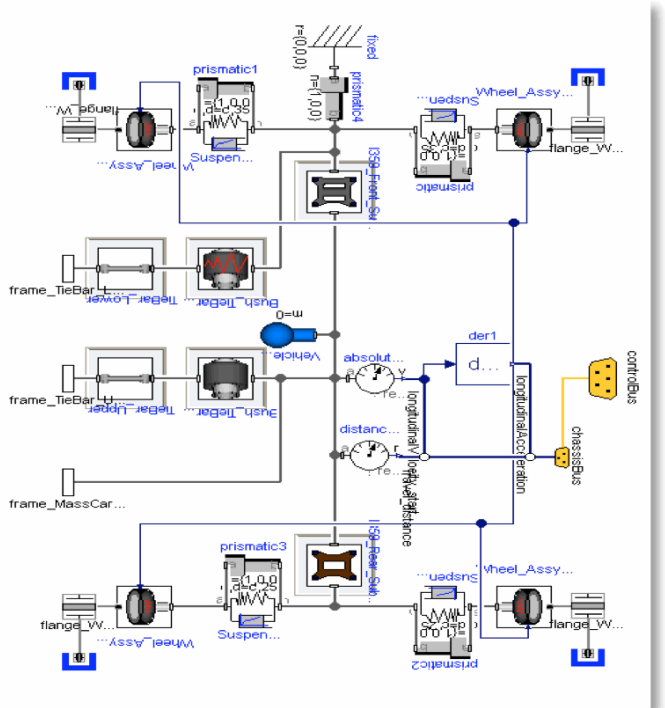

Figure 12. Compliant 4wd driveline [3]

Mass models of vehicles can be created with stiffness and damping properties between major components and their mounting to the chassis in order to investigate secondary ride effects. Again this could prove invaluable for investigating the ride comfort of current and future hybrid vehicles, or even for deeper investigation of the environment of developing hybrid technologies, such as battery packs. 
Furthermore through development of different instances of component models, such as a mass model with rigid or compliant mounting to chassis, the same model can be utilised for all required studies, complexity can be reduced for pure handling manoeuvres by utilising reduced order component models, and complexity can be increased for primary and secondary ride investigations, by simply replacing the required component models. What's more, the fidelity can be given to required areas of the model, for instance if requirements dictate, a very high DOF drivetrain model can be utilised with a simplified chassis model, such an approach allows model fidelity where needed but also gives the ability to improve run times by reducing complexity in other areas.

\section{CONCLUSION}

The development of a multibody chassis model in Dymola has been illustrated. The model has been validated for use in a specific ride and handling investigation. However whilst its validation and usage form part of a specific study, it is envisaged that the modelling process discussed can be used as a basis to create a joined up approach to ride, handling and driveability studies for current and future HEV's.

It was shown that through the modular modelling approach used here, that a base model can be easily adapted and extended to fit any number of hybrid architectures, furthermore model fidelity can easily be adjusted in the same way. Such an approach could lead to greater efficiency in modelling of HEV's, and a more joined up approach between the current powertrain and control systems studies and vehicle dynamics studies in HEV's.

\section{REFERENCES}

[1] Bastin, M. (2015). An Investigation into the Effects of Hybrid Electric Vehicle Powertrains on Ride and Handling. Ph.D. Thesis, University of Warwick.

[2] Bastin, M. \& Jones, R., P. (2013). The effects on ride and handling due to modified mass distribution arising in hybrid electric vehicles. In: Vehicle Dynamics and Control, Cambridge University.

[3] Bastin, M., Shah, M., Cheng, C. \& Jones, R., P. . (2011). Physical modelling of vehicle systems dynamics for drivability and handling studies. In: Vehicle Dynamics and Control, Cambridge University.

\section{AUTHORS}

Matthew Bastin* and R Peter Jones are with School of Engineering, University of Warwick, Coventry, UK (* corresponding author: e-mail matt.a.bastin@gmail.com).

Submitted 13 September 2015. Published as resubmitted by the authors 23 October 2015. 